\title{
Locus de Controle Materno, Satisfação com a Vida e Relacionamento com o Filho Adolescente ${ }^{1}$
}

\author{
Cristina Maria de Souza Brito Dias ${ }^{2}$ \\ Universidade Católica de Pernambuco \\ Bartholomeu Torres Tróccoli \\ Universidade de Brasília
}

\begin{abstract}
RESUMO - Foram investigadas influências do nível de satisfação com a vida e controle materno sobre a percepção mantida por mães, quanto aos relacionamentos com seus filhos adolescentes. Duzentas e cinqüenta e seis mães responderam a um único questionário, contendo as variáveis de interesse da pesquisa. Além dos índices gerais, foram computados resultados de quatro dimensões subjacentes à escala satisfação com a vida e cinco subjacentes à escala controle parental. A percepção do grupo de mães sobre seus relacionamentos com filhos adolescentes também foi mensurada por um índice geral e cinco dimensões específicas. Análises de regressão múltipla hierárquica revelaram que, mesmo controlando a influência do conjunto de variáveis demográficas, uma quantidade significativa da variância do relacionamento mãe-filho adolescente pode ser predita pelos índices gerais das variáveis satisfação com a vida e locus de controle parental, bem como pelos índices específicos das dimensões controle parental, responsabilidade parental e eficácia parental.
\end{abstract}

Palavras-chave: relação mãe-filho adolescente; controle maternal; satisfação com a vida.

\section{Maternal Locus of Control, Life Satisfaction and the Perception of the Mother-Adolescent Child Relationship}

\begin{abstract}
This study investigated the influence of life satisfaction and maternal locus of control on the perception of mothers about their relationships with their adolescent child. Two hundred fifty-six mothers answered a questionnaire with all the relevant dimensions of the study. Besides of the general scores, results of four specific dimensions underlying the life satisfaction scale and five dimensions underlying the locus of parental control scale were analyzed. The mothers' perception about their relationship with their adolescent children was also measured through a general score and five specific dimensions. Even after the control of the amount of demographic variance, hierarchical multiple regression analysis showed that a significant amount of variance of mother-adolescent children relationship may be predicted by the general measures of life satisfaction, parental locus of control and also by the specific measures of factors like parental control, parental responsibility and parental efficacy.
\end{abstract}

Key words: mother-adolescent child relationship; maternal control; life satisfaction.

Entre as questões e tarefas que a maioria das pessoas adultas se defronta estão a escolha de uma profissão, a convivência com um(a) parceiro(a) e a criação de filhos. Tudo isso envolve problemas e decisões que estão interligados e exercem mútua influência (Glenn \& McLanahan, 1982; Koski \& Steinberg, 1990; Mac Dermid, 1990; Olson, McCubbin, Barnes, Larson, Muxen \& Wilson, 1989; Ribeiro, 1992).

Uma vez que os laços que unem pais e filhos são difíceis de romper e a freqüência do contato entre eles é grande, ao menos até que o filho adquira sua independência, o conteúdo dessa relação é especialmente relevante para o bem-estar

1 Este trabalho é derivado da Tese de Doutorado "Relacionamento com Filho Adolescente: Percepção de Mães", elaborada pela primeira autora, sob a orientação do segundo autor. Os autores agradecem ao CNPq pelo apoio concedido, por meio de bolsa de doutorado, para a primeira autora.

2 Endereço: Rua Bernardo Vieira de Melo, 105. CEP: 53020-160 Olinda -PE. dos pais (Umberson, 1989). Em um sentido mais estrito, o bem-estar dos pais pode ser definido como sendo a satisfação que os membros do casal sentem individualmente com relação à vida e ao casamento.

A satisfação com a vida pode ser definida como a relação de satisfação harmoniosa entre aquilo que o indivíduo desejou e alcançou, ou como a preponderância do afeto positivo sobre o negativo (Diener, 1984). Vários fatores contribuem para o nível de satisfação com a vida: casamento, contato social, emprego, eventos da vida, família, idade, influência biológica, nível educacional, personalidade, raça, religião, residência, transporte (Diener, Larson, 1978).

Quanto ao casamento, vida em família e satisfação conjugai, encontram-se evidências nas pesquisas de que constituem os mais fortes preditores do bem-estar humano (Glenn \& Weaver, 1981; Wilson, 1967). Bengtson, Rosenthal e Burton (1990) salientaram que, comparadas às pessoas solteiras, as casadas apresentam os mais altos níveis de saúde física, mental e moral, satisfação com a vida, apoio e integração social, recursos econômicos e menores índices de institucionalização. 


\section{M. S. Brito Dias e B.T. Tróccoli}

Alguns dos fatores que influenciam a satisfação conjugal também estão envolvidos, direta ou indiretamente, com a satisfação com a vida. Entre os fatores que afetam significativamente a satisfação conjugai, encontra-se a influência negativa dos filhos (Rollins \& Galligan, 1978, citado em Brehm, 1985). Outros estudos, entretanto, mostram que a presença dos filhos diminui o risco do rompimento conjugai (Morgan \& Rindfuss, 1985; Waite, Haggstrom \& Kanouse, 1985). Abbot e Brody (1985) consideram injustificado afirmar que todas as crianças diminuem o ajustamento conjugai, mas admitem que tal diminuição pode estar mediada por variáveis como o gênero, a idade e a quantidade de filhos.

Talvez seja o caso de se considerar que as influências dos filhos variam de acordo com diferentes momentos da vida do casal. Harris, Ellicott e Holmes (1986) argumentam que esta relação deve ser analisada em função de seis fases do ciclo de vida familiar: fase sem crianças, fase pré-escolar, fase da escola, fase da adolescência, fase da saída de casa, fase pós-parental. A estas seis fases foi acrescentada uma sétima fase, a da aposentadoria (Olson \& cols., 1989). Relacionando o ciclo de vida familiar com a satisfação conjugal, estudos demonstram um decréscimo gradual na satisfação com o casamento e uma tendência curvilinear, onde a satisfação decresce nos primeiros estágios, segue-se uma fase de nivelamento para depois haver um aumento da satisfação nas fases posteriores do ciclo familiar (Anderson, Russell \& Schumm, 1983; Rollins \& Cannon, 1974; Rollins \&Feldman, 1970; Troll, 1975; Olson \& cols.).

Embora os laços entre pais e filhos continuem por toda a vida, existem fases em que eles são postos mais à prova, e a adolescência parece constituir-se em uma delas. Tamir e Antonucci (1981), em uma pesquisa com 2000 adultos, em diversos estágios do ciclo de vida familiar, concluem que o estágio dos filhos adolescentes é o mais problemático. Olson e cols. (1989) também argumentam que os anos da adolescência são os mais estressantes para os pais, porque ambos vivem em mundos diferentes.

Estudos feitos sobre a adolescência identificaram dois grandes tópicos relacionadas à referida fase: o primeiro, de que é uma fase de tumulto e estresse; e o segundo, que se refere ao distanciamento entre pais e filhos, dada a preferência pelo grupo de companheiros (Greenberg, Siegel \& Leitch, 1982). No entanto, outras pesquisas têm demonstrado que a adolescência é um período de mudança gradual, que não é definido nem pela instabilidade, nem pela regressão do ego. Os pais também continuam a desempenhar papel importante na vida do adolescente, não sendo antagônicos aos companheiros do mesmo (Dornsbuch, 1989; Fasick, 1984; Fleming, 1993; Gecas \& Seff, 1990; Lopes, 1994; Montemayor \& Brownlee, 1987; Waldemar, 1983).

Para Fleming (1993) e Sampaio (1994), a questão central na relação pais-filhos adolescentes refere-se à autonomia destes. O controle parental, portanto, parece ser especialmente importante durante a fase dos filhos adolescentes. Ele compreende a maneira pela qual os pais inibem o comportamento do filho e envolve várias estratégias como: proteção, supervisão, clareza nas regras, punição, coerção, disciplina, restrição, asserção de poder, permissividade e indução (Gecas $\&$ Seff, 1990).

A questão do controle parental pode ser enfocada a partir do quadro referencial da Teoria do Locus de Controle de Rotter (Lefcourt, 1991). De acordo com esta teoria, as pessoas de maior internalidade são aquelas que acreditam que as conseqüências dos seus comportamentos são contingentes às suas ações. Já as pessoas de maior externalidade acreditam que as consequiências dos seus comportamentos estão sempre na contingência de fatores externos e não sob o próprio controle.

Diversas escalas já foram elaboradas para avaliar o locus de controle dos indivíduos de acordo com os mais diversos campos: saúde, funcionamento sexual, velhice, trabalho, satisfação conjugal, competência, comportamento afiliativo e de realização, controles interpessoal e sócio-político, realização nos esportes e cuidado com os filhos. Quanto ao controle parental, foi desenvolvida a escala de Locus de Controle Parental, procurando avaliar a extensão na qual os pais acreditam que podem moldar o comportamento e o futuro da vida dos filhos (Furham, 1995). Campis, Lyman e PrenticeDunn (1986) tinham sugerido, por exemplo, que a orientação externa, no aspecto do controle parental, estaria relacionada com sentimentos de incompetência e falta de controle sobre os filhos.

As pesquisas sobre a família e a adolescência têm-se caracterizado por uma certa preocupação com o contexto social que cerca o adolescente, deixando de lado aspectos do seu desenvolvimento individual. Entretanto, os estudos nesta área ainda são poucos, principalmente no que diz respeito às influências recíprocas entre pais e filhos, às relações entre pais e filhos do mesmo sexo e do sexo oposto, bem como estudos longitudinais que permitam sugerir alguma relação de causalidade entre as variáveis analisadas (Gecas \& Seff, 1990).

A relevância de construtos como satisfação com a vida, satisfação conjugal e locus de controle parental, sugere que o estudo dos seus possíveis inter-relacionamentos pode contribuir substancialmente para a compreensão das relações pais-filhos adolescentes. No Brasil, a população compreendida entre os 10 e 14 anos, portanto vivenciando a puberdade, totaliza 170.047.150 milhões e na faixa de idade entre os 15 e 19 anos são 150.017.631 milhões de indivíduos, o que realça mais ainda a importância desses estudos (IBGE, 1991).

Esta pesquisa procurou, portanto, verificar possíveis influências da satisfação com a vida e locus de controle maternal sobre a percepção que as mães possuem acerca dos seus relacionamentos com seus filhos adolescentes.

\section{Método}

\section{Amostra}

Participaram da pesquisa 256 mães de adolescentes cujo(a) filho(a) mais velho(a) estava na faixa etária entre 13 e 18 anos. As mães residiam, em sua maioria, no Plano Pilo- 
to da cidade de Brasília, embora fossem provenientes de todas as regiões brasileiras. Todas as mulheres exerciam uma atividade remunerada e apresentavam, no período da pesquisa, idade média de 41 anos, com desvio padrão de 4 anos e 3 meses. A média de idade dos filhos foi de 16 anos, com desvio padrão de 1 ano e 7 meses, sendo que 130 eram do sexo masculino, 124 do sexo feminino e 2 participantes não indicaram o sexo do filho.

Quanto ao estado civil das respondentes, 187 eram casadas, 11 recasadas, 41 separadas, desquitadas ou divorciadas, 10 solteiras e 7 viúvas. Em relação à escolaridade, 185 tinham curso superior e 71 curso médio. Para fins de análise dos dados, foram consideradas, no grupo de casadas, as 187 casadas e as 11 recasadas, totalizando 198 mulheres. As demais foram consideradas como participantes do grupo das descasadas, totalizando 58 mulheres.

\section{Instrumentos}

Foi aplicado um único questionário geral incorporando as dimensões envolvidas na questão mãe-filho adolescente. O instrumento Dimensões do Relacionamento Mãe-Filho (DREMAF) constou de itens que tiveram como ponto de partida traduções e adaptações de duas escalas (descritas a seguir), bem como de itens especialmente elaborados com o objetivo de verificar a percepção do relacionamento mãefilho. A tradução livre das escalas originais foi submetida a duas pessoas com bom conhecimento da língua inglesa, que realizaram nova tradução para o inglês. A adequação entre as duas versões foi analisada pela pesquisadora e tradutoras, resultando na versão final em português. $\mathrm{O}$ instrumento completo foi aplicado a 14 mães, com a finalidade de detectar afirmações que ainda apresentavam dificuldades de compreensão. A parte da versão final do DREMAF utilizada na presente pesquisa compreendeu as seguintes escalas:

(1) Escala de Satisfação com a Vida - Esta escala foi traduzida da Life Satisfaction Scale - Index (LSIA), sendo uma das duas formas alternativas da Life Satisfaction Scale (LSR) desenvolvida por Neugarten, Havighurst e Tobin (1961). Originalmente a LSR destinou-se a mensurar cinco fatores constituintes da satisfação com a vida em geral, mas outros autores também identificaram diferentes estruturas fatoriais na LSI-A (Adams, 1969; Hoyt \& Creech, 1983; Lohmann, 1980). Estudos realizados pelos autores indicaram correlações significativas da LSI-A com a LSR $(r=0,55 ; p<0,01)$ e também com classificações realizadas por psicólogos clíni$\cos (\mathrm{r}=0,39 ; p<0,01)$.

Uma única modificação foi introduzida na escala LSI-A. Como um dos itens da escala original ("Já sinto minha idade e ela não me aborrece") abrange dois conceitos, ele foi desdobrado em dois na escala a ser utilizada ("Já sinto minha idade"; "Minha idade não me aborrece"). A escala a ser utilizada ficou então com 21 itens, com os quais o respondente podia concordar, discordar ou ficar em dúvida.

Os 21 itens do DREMAF referentes à versão empregada da escala LSI-A foram submetidos a uma análise fatorial (ver Dias, 1995), resultando na identificação de um fator geral de segunda ordem (Satisfação Geral com a Vida) e quatro fatores de primeira ordem: Congruência ("eu não mudaria meu passado, mesmo que pudesse"; "eu tenho obtido basicamente tudo que esperava da vida"); Animação vs. Apatia ("a maioria das coisas que faço são monótonas e chatas"; "quando penso em minha vida vejo que não consegui muitas das coisas que desejei"); Autoconceito ("já sinto a minha idade"; "a minha idade não me aborrece") e Humor ("eu me sinto tão feliz como me sentia quando era mais jovem"; "estes são os melhores anos de minha vida"). Os quatro fatores de primeira ordem foram identificados, em grande parte, como semelhantes aos encontrados por Adams (1969) e Hoyt e Creech (1983) para a versão original da LSI-A.

(2) Escala de Locus de Controle Parental - Esta escala foi obtida com a tradução da escala Parental Locus of Control (PLOC), de Campis e cols. (1986), desenvolvida para medir o grau de controle dos pais sobre os filhos em geral. A PLOC apresentou correlação positiva e significativa com a Escala Locus de Controle Geral I-E de Rotter.

Dias, Tróccoli e Dias (1996) realizaram uma análise fatorial dos 47 itens da escala Locus de Controle Parental, encontrando dois fatores de segunda ordem e cinco fatores de primeira ordem. Os dois fatores de segunda ordem correspondem aos conceitos de internalidade e externalidade (Lefcourt, 1991). Já os cinco fatores de primeira ordem foram: (1) Eficácia Parental (medindo o quanto os pais se sentem efetivos no papel parental: "o que eu faço tem muito efeito sobre o comportamento do meu filho adolescente"; "quando alguma coisa vai mal entre eu e meu filho adolescente, posso fazer muita coisa para corrigir esta situação"); (2) Controle Parental (que avalia o quanto os pais se sentem capazes de controlar o comportamento dos filhos: "o comportamento do meu filho é, algumas vezes, mais do que posso suportar"; "às vezes eu sinto que não tenho controle suficiente sobre o rumo que a vida do meu filho adolescente está tomando"); (3) Incompetência Parental (avaliando o quanto os pais se consideram com pouca competência para desempenhar seus papéis: "pessoas capazes podem não ser bons pais por não terem aproveitado bem as oportunidades que surgiram"; "os problemas de comportamento dos filhos são, freqüientemente, devidos a erros que os pais cometem"); (4) Crença na Sorte ou Destino (compreendendo a crença parental que o comportamento do filho é influenciado por fatores externos: "ser uma boa mãe depende, freqüentemente, de ter sorte bastante para ter um bom filho"; "a hereditariedade desempenha o principal papel na determinação da personalidade dos filhos"); e (5) Responsabilidade Parental (indicando o quanto os pais se sentem responsáveis pelo comportamento do filho: "não existem filhos bons ou maus apenas bons ou maus pais"; "os problemas de comportamento do meu filho não são culpa de ninguém, mas minha"). Apenas um dos fatores da PLOC original, Controle pelos Filhos, não foi observado no estudo fatorial de Dias e cols.. Em seu lugar, surgiu a dimensão Incompetência Parental. 


\section{M. S. Brito Dias e B.T. Tróccoli}

(3) Percepção e Avaliação do Relacionamento Mãe-Filho Adolescente - Esta dimensão foi mensurada por meio de 15 questões, com várias subdivisões, sendo que a participante podia concordar, discordar ou ficar em dúvida com as mesmas (Dias, 1995). Estas 15 questões foram derivadas de uma Análise de Conteúdo das respostas dadas a uma entrevista realizada com 20 mães de adolescentes. Para esta pesquisa foram utilizados os resultados obtidos com oito das quinze questões originais, reagrupadas em torno das seguintes dimensões: Vida profissional da mãe; Preocupações maternas; Sentimentos maternos; Futuro materno e Pontos positivos de ter filho adolescente.

Finalmente, foram incluídas no DREMAF questões sobre a idade, naturalidade, escolaridade, profissão, estado civil, tempo de casamento, número de filhos, sexo e idade dos mesmos.

\section{Procedimentos}

Mulheres com educação equivalente ao segundo grau ou mais e o(a) filho(a) mais velho(a) na faixa dos 13 aos 18 anos foram procuradas em escolas, ambientes de trabalho e vizinhança, e lhes foi explicado que se tratava de uma pesquisa sobre o relacionamento com o filho adolescente. Devido à extensão do questionário, algumas participantes não puderam responder no momento da abordagem, sendo-lhes permitido levá-lo para casa e devolvê-lo posteriormente. Os questionários respondidos eram colocados em envelopes, junto a outros, de forma a não permitir a identificação da respondente.

\section{Resultados}

Foram realizadas análises de regressão múltipla hierárquica, com o objetivo de verificar se os resultados obtidos, quanto à percepção e avaliação do relacionamento mãe-filho adolescente, podem ser preditos a partir de algumas variáveis demográficas e dos índices gerais e específicos da satisfação com a vida e locus de controle parental.

Dois conjuntos de variáveis foram utilizados nestas análises de regressão múltipla hierárquica. Em primeiro lugar, foi introduzido na equação de regressão o conjunto de variáveis demográficas (tempo de casamento, sexo do filho, estado civil, número de filhos, idade da participante, idade do filho e duas variáveis dummy representativas de três níveis da variável grau de instrução: médio, superior incompleto e superior completo), seguido pelo conjunto dos índices gerais das variáveis independentes satisfação com a vida e locus de controle parental ou dos índices específicos (fatores) destas mesmas variáveis.

A questão da pesquisa foi a seguinte: se as informações quanto às diferenças nas variáveis satisfação com a vida e locus de controle parental podem ser usadas para predizer a percepção do relacionamento com o(a) filho(a) adolescente, após as diferenças nas variáveis demográficas terem sido eliminadas estatisticamente.
A Tabela 1 apresenta os principais resultados das duas regressões do conjunto de variáveis demográficas (primeira linha), e dos índices gerais (segunda linha) e específicos (terceira linha) das variáveis satisfação com a vida e locus de controle parental sobre o índice geral da variável percepção do relacionamento mãe-filho. Na primeira análise de regressão hierárquica, foram introduzidas, em primeiro lugar, as variáveis demográficas e, em segundo lugar, os índices gerais das variáveis satisfação com a vida e locus de controle parental. Na segunda análise de regressão hierárquica, o conjunto de variáveis demográficas foi novamente introduzido em primeiro lugar sendo seguido, no entanto, pelos índices específicos (dimensões fatoriais) das variáveis satisfação com a vida e locus de controle parental. Na segunda coluna da Tabela 1, estão as correlações semi-parciais entre cada variável preditora e a variável dependente, bem como a média e o desvio padrão da variável dependente (VD). Na terceira coluna, encontram-se os incrementos na predição da variância da VD, obtidos após a introdução de cada bloco de variáveis, isto é, as mudanças nos coeficientes de correlação múltipla decorrentes do acréscimo do último conjunto de variáveis. Pode-se observar na Tabela 1 que o primeiro incremento não foi significativo $\left(R^{2} \underset{\text { mudança }}{,}=0,19=0,02\right)$, enquanto que os outros dois incrementos representaram acréscimos significativos à variância explicada pelos índices demográficos: $R^{2} \underset{\text { mudança }}{,}=0,11$ devido aos índices gerais e $R^{2}$ $=0,20$ devido aos índices específicos das variáveis mudança

satisfação com a vida e locus de controle parental. A última coluna da Tabela 1 apresenta o coeficiente de regressão múltipla $(R)$, o coeficiente de regressão múltipla ao quadrado $\left(R^{2}\right)$ e o coeficiente de regressão múltipla ao quadrado ajustado para o tamanho da amostra ( $R^{2}$ ajustado) obtidos

Tabela 1 - Regressão Hierárquica do conjunto de variáveis demográficas, satisfação geral com a vida, controle parental geral e fatores específicos dessas duas últimas variáveis, sobre o índice geral da percepção do relacionamento com o filho adolescente.

\begin{tabular}{|c|c|c|c|}
\hline Variáveis & $\begin{array}{l}\text { Correlaçōes semj- } \\
\text { parciais com a } \\
\text { variáve! relłaçăo } \\
\text { màe-fillo (VD) }\end{array}$ & $\begin{array}{l}\text { Mudança no } R^{2} \\
\text { (incremento) }\end{array}$ & 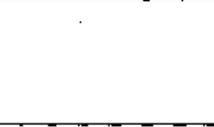 \\
\hline Dethografficas & $\cdot$ & 0,02 & \\
\hline Sutisfaçăo geral & 0,19 ** & & \\
\hline Controle parental geras & $0,22 * *$ & $0,1 !^{\text {*t* }}$ & $R^{2}=0,13$ \\
\hline Média & 2,27 & & $R^{2}$ (ajustado) $=0,09$ \\
\hline Desvio Padräo & 0,16 & & $R=0,36 * *$ \\
\hline Congruência & 0,06 & & . \\
\hline Aninaçāo vs. Apatia & $0,0 \mathrm{l}$ & & \\
\hline Autoconceito & $0,12^{*}$ & & \\
\hline Humor & $-0,02$ & & \\
\hline Eficácia parental & 0.03 & & \\
\hline Controle parental & $0,27 *$ & & \\
\hline Incompetência parental & 0,01 & & \\
\hline Sorte ou destine & $.0,07$ & & \\
\hline Responsabilidade & $0.12^{* *}$ & $0,20^{* *}$ & $R^{2}=0,22$ \\
\hline \multirow[t]{2}{*}{ parental } & & & $R^{*}($ ajustado $)=0,16$ \\
\hline & & & $R=0,47^{* *}$ \\
\hline
\end{tabular}

$*<<0.05 ; * *<<0.001$ 
no último passo de cada uma das duas análises de regressão hierárquica.

Os resultados da Tabela 1 indicam que o conjunto das variáveis demográficas não predizem uma quantidade significativa de variância no índice geral da percepção do relacionamento mãe-filho. Entretanto, uma vez eliminadas estatisticamente as diferenças entre as participantes quanto às características demográficas, verifica-se que as variáveis satisfação geral com a vida e locus de controle parental geral estão significativamente relacionadas com a percepção e avaliação do relacionamento mãe-filho. Entre os índices específicos, apenas a dimensão autoconceito (aumento na percepção da idade e do envelhecimento) e as dimensões controle parental (o quanto os pais se sentem capazes de controlar o comportamento dos filhos) e a responsabilidade parental (o quanto os pais se sentem responsáveis pelos comportamentos dos filhos) contribuíram significativamente para o total da variância explicada.

Os resultados da Tabela 1 indicam que uma maior satisfação geral com a vida e a percepção de maior controle em geral sobre os filhos, estão associados positivamente com a qualidade da percepção geral do relacionamento mãe-filho. Considerando apenas as dimensões específicas, verifica-se que uma maior consciência do próprio envelhecimento, um sentimento de maior responsabilidade pelo comportamento dos filhos e maior capacidade para controlar os filhos, permitiram prever uma porção significativa da avaliação que as mães fizeram sobre seus relacionamentos em geral com os filhos adolescentes.

A Tabela 2 apresenta resultados de análises semelhantes, exceto pela mudança na variável dependente que passa a ser uma das dimensões da escala de percepção do relacionamento mãe-filho (vida profissional da mãe). Considerando a opinião das participantes quanto a vida profissional, os resultados da Tabela 2 mostram resultados bem próximos dos reproduzidos na Tabela 1 , exceto que desta vez o conjunto de variáveis demográficas obteve um incremento significativo na predição das opiniões sobre a vida profissional $\left(R_{\text {mudança }}^{2}=0,07\right)$. Contribuiu significativamente para este resultado a variável estado civil. O restante dos resultados da Tabela 2 indica também que ocorreu, nas dimensões específicas, apenas a significância isolada da dimensão controle parental.

Estes resultados mostram que tanto o estado civil quanto a satisfação geral com a vida e o controle parental geral permitiram prever significativamente a avaliação que as mães fizeram sobre suas vidas no trabalho. Há uma associação positiva entre uma maior satisfação com a vida e um maior controle parental com uma percepção positiva da influência dos filhos na vida profissional.

Os resultados obtidos com os sentimentos maternos, como variável dependente, estão na Tabela 3. Verifica-se, nesta tabela, que todos os conjuntos de variáveis apresentaram predições significativas com esta dimensão da percepção do relacionamento mãe-filho, acrescentando-se ainda a dimensão eficácia parental (o quanto os pais se sentem efetivos no papel parental), a dimensão controle parental entre
Tabela 2 - Regressão Hierárquica do conjunto de variáveis demográficas, satisfação geral com a vida, controle parental geral e fatores específicos dessas duas últimas variáveis sobre o fator vida profissional das mães.

\begin{tabular}{|c|c|c|c|}
\hline Variáveis & $\begin{array}{l}\text { Correlações semi- } \\
\text { parciais com a } \\
\text { variável vida } \\
\text { profissional da } \\
\text { mãe (VD) }\end{array}$ & $\begin{array}{l}\text { Mudança no } R^{2} \\
\text { (incremento) }\end{array}$ & \\
\hline Demográficas & - & 0,02 & \\
\hline Estado civil & $0,22 *$ & $0,07 *$ & \\
\hline Satisfação geral & $0,16^{*}$ & & \\
\hline Controle parental geral & $0,14^{*}$ & $0,06^{* *}$ & $\mathrm{R}^{2}=0,12$ \\
\hline Média & 2,14 & & $R^{2}$ (ajustado) $=0,09$ \\
\hline Desvio Padrão & 0,33 & & $R=0,35^{* *}$ \\
\hline Congruência & 0,09 & & \\
\hline Animação vs. Apatia & 0,07 & & \\
\hline Autoconceito & $-0,01$ & & \\
\hline Humor & $-0,01$ & & \\
\hline Eficácia parental & $-0,01$ & & \\
\hline Controle parental & $0,13 *$ & & \\
\hline Incompetência parental & 0,02 & & \\
\hline Sorte ou destino & $-0,01$ & & \\
\hline Responsabilidade & 0,03 & $0,08 *$ & $\mathrm{R}^{2}=0,14$ \\
\hline \multirow[t]{2}{*}{ parental } & & & $R^{2}$ (ajustado) $=0,08$ \\
\hline & & & $R=0,38 *$ \\
\hline
\end{tabular}

$* p<0,05 ; * * p<0,001$.

as dimensões específicas com predição significativa desta variável dependente. Quanto às variáveis demográficas, contribuíram significativamente as variáveis estado civil e tempo de casamento.

Sentimentos positivos para com os filhos adolescentes foram previstos por uma maior satisfação geral com a vida, por um controle parental maior, bem como pelos índices específicos da eficácia parental e do controle parental. Pela primeira vez, o tempo de casamento contribuiu significativamente para a previsão de um aspecto da relação mãe-filho. Esta previsão, no entanto, ocorreu na direção negativa: quanto mais tempo de casamento, menos sentimentos positivos relativos ao fato de ser mãe de filho adolescente.

Finalmente, as variáveis e dimensões analisadas na pesquisa também permitem uma predição significativa da quantidade de pontos positivos percebidos na relação mãe-filho (Tabela 4). Uma vez eliminada a contribuição (não significativa) das variáveis demográficas, observa-se na Tabela 4 que tanto os índices gerais quanto específicos predizem, no seu conjunto, significativamente esta dimensão da relação mãefilho. Individualmente, entretanto, os índices gerais da satisfação com a vida e do locus de controle parental não contribuem de forma significativa para a predição da percepção dos pontos positivos na relação mãe-filho. Já nas dimensões específicas, observa-se novamente a contribuição significativa da dimensão controle parental acompanhada da dimensão responsabilidade parental (o quanto os pais se sentem responsáveis pelo comportamento do filho). Os índices indicativos da percepção de um maior controle e de uma maior responsabilidade pelo comportamento dos filhos permitiram a previsão significativa de mais pontos positivos em ser mãe de filho adolescente. 
Tabela 3 - Regressão Hierárquica do conjunto de variáveis demográficas, satisfação geral com a vida, controle parental geral e fatores específicos dessas duas últimas variáveis sobre o fator sentimentos maternos.

\begin{tabular}{|c|c|c|c|}
\hline Variäveis & $\begin{array}{l}\text { Correlaçòes semi- } \\
\text { parciais com a } \\
\text { vanível } \\
\text { sentimentos } \\
\text { matemos (VD) }\end{array}$ & $\begin{array}{l}\text { Mudança no } R^{1} \\
\text { (incremento) }\end{array}$ & \\
\hline \multicolumn{4}{|l|}{ Demográficas } \\
\hline Estado civil & $0.13^{*}$ & $0,08 *$ & \\
\hline Tempo de casamento & $-0,20^{*}$ & & \\
\hline Satistaçāo geral & $0,14 *$ & & \\
\hline Controle parental geral & $0,42^{* *}$ & $0,23^{* *}$ & $R^{2}=0,30$ \\
\hline Média & 2,34 & & $R^{2}$ (ajustado $)=0,28$ \\
\hline Desvio Padrâo & 0.32 & & $R=0,55^{* *}$ \\
\hline 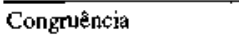 & 40,01 & & \\
\hline Animaçâo vs. Apatia & 0,06 & & \\
\hline Auloconceito & 0,05 & & \\
\hline Humor & $-0,01$ & & \\
\hline Eficácia parental & $0,11^{*}$ & & \\
\hline Controle parental & $0,35^{* *}$ & & \\
\hline lacompetência parenta] & 0,05 & & \\
\hline Sorte ou destino & 0,01 & & \\
\hline Responsabilidade & 0,07 . & $0,31 * *$ & $R^{2}=0,38$ \\
\hline \multirow[t]{2}{*}{ parental } & & & $R^{2}($ ajustado $)=0,34$ \\
\hline & & & $R=0,62^{* * *}$ \\
\hline
\end{tabular}

$* p<0.05 ; * p<0,001$

Tabela 4 - Regressão Hierárquica do conjunto de variáveis demográficas, satisfação geral com a vida, controle parental geral e fatores específicos dessas duas últimas variáveis sobre o fator pontos positivos na relação mãe-filho.

\begin{tabular}{|c|c|c|c|}
\hline Variáveis & $\begin{array}{l}\text { Correlaçōes semu- } \\
\text { parciais com a } \\
\text { variável pontos } \\
\text { positivos de ter } \\
\text { fllho adolescente } \\
\text { (VD) }\end{array}$ & $\begin{array}{l}\text { Mudança no } R^{2} \\
\text { (incremento) }\end{array}$ & 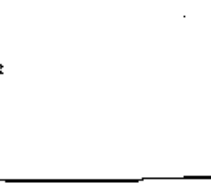 \\
\hline Demográficas & & 0,04 & \\
\hline Satisfaçăo geral & 0,11 & & \\
\hline Controk parental geral & 0,12 & $0,03^{*}$ & $R^{1}=07$ \\
\hline Médias & 2,54 & & $R^{1}$ (ajustado) $=0,03$ \\
\hline Desvios Padrâo & 0,38 & & $R=0,27 * *$ \\
\hline Congruência & 0.04 & & \\
\hline Animaçāo vs. Apatia & $-0,09$ & & \\
\hline Autoconceito & 0,06 & & \\
\hline Humer & 0.07 & & \\
\hline Eficácia parental & $-0,01$ & & \\
\hline Controle parental & $0,16^{*}$ & & \\
\hline Incompetēncia parental & 0,01 & & \\
\hline Sorte ou destino & $-0,04$ & & \\
\hline Responsabilidade & $0,15^{*}$ & $0,10^{*}$ & $R^{2}=14$ \\
\hline parental & & & $\begin{array}{l}R^{2} \text { (ajustado) }=0,08 \\
R=0.38^{* *}\end{array}$ \\
\hline
\end{tabular}

$* p<0.05$

Duas das dimensões da percepção e avaliação da relação mãe-filho não foram preditas de forma significativa pelas variáveis satisfação com a vida e locus de controle parental, uma vez eliminada a contribuição das variáveis demográficas. Nenhuma predição significativa foi observada com o fator preocupações maternas. Já o fator relativo às expectativas quanto ao próprio futuro, foi predito significativamente apenas pelo conjunto total de variáveis demográficas $(R=.24 ; p$ $<0,05)$, com o estado civil contribuindo significativamente $(s r=.18 ; p<0,05)$. Nenhuma das duas variáveis estudadas apresentou incrementos significativos para além das variáveis demográficas nestes dois aspectos do relacionamento mãe-filho.

Considerando os resultados das análises de regressão, foram feitas análises adicionais para uma melhor descrição dos resultados obtidos em cada um dos três principais conjuntos de variáveis estudados, bem como para analisar melhor as diferenças que surgiram devidas ao estado civil das participantes.

Para cada um dos fatores de primeira ou segunda ordem das escalas de satisfação com a vida, locus de controle parental e percepção e avaliação do relacionamento mãefilho, foram calculadas as médias das participantes casadas e descasadas. Estes resultados estão na Tabela 5.

\section{Satisfação com a vida}

A análise de variância mista, entre o estado civil (variável entre-grupos) e os fatores da satisfação com a vida (variável com medidas repetidas intra-grupos), demonstrou a existência de diferenças significativas para o estado civil $\left[F_{(1,226)}=8,47 ; p<0,004\right]$ e a satisfação com a vida $\left[F_{(1,3)}=\right.$ $41,09 ; p<0,001]$. Os resultados revelaram ainda ausência de efeitos de interação entre o estado civil e as medidas de satisfação com a vida. Em todos os fatores, as mulheres casadas apresentaram índices mais elevados que as mulheres descasadas. Como grupo, as mulheres casadas sentiram-se mais congruentes ("não mudaria meu passado"), mais animadas ("as coisas que faço não são monótonas e chatas"), com melhor autoconceito ("a minha idade não me aborrece") e com um humor um pouco mais positivo ("estes são os melhores anos da minha vida"), que as mulheres descasadas. Considerando apenas os fatores representativos da satisfação com vida, as mulheres em geral tenderam a concordar mais com as questões do autoconceito $(=2,6)$ seguido por Animação/Apatia $(=2,5)$, Congruência $(=2,2)$ e Humor $(=2,1)$.

\section{Locus de controle parental}

Tendo por base os resultados da análise fatorial realizada por Dias e cols. (1996), foram calculados cinco índices para cada participante, correspondendo a cada um dos fatores de primeira ordem, bem como um índice geral representando o Locus de Controle Parental Geral. A Tabela 5 também apresenta os resultados médios nos escores fatoriais obtidos com os fatores de primeira ordem da escala locus de controle parental.

Uma análise de variância mista, entre o estado civil (variável independente entre-grupos) e o locus de controle parental (variável independente intra-grupos), revelou a existência de diferenças significativas para o estado civil $\left[F_{(1,206)}\right.$ $=13,79 ; p<0,001]$ e locus de controle parental $\left[F_{(1,4)}=\right.$ 
Tabela 5 - Médias e desvios-padrão dos escores fatoriais resultantes das Escalas de Satisfação com a Vida, Locus de Controle Parental e Percepção e Avaliação do Relacionamento Mãe-Filho Adolescente, de acordo com o estado civil.

\begin{tabular}{|c|c|c|c|c|c|}
\hline $\begin{array}{l}\text { Escala de (a) } \\
\text { Satisfação com a vida }\end{array}$ & Congruência & Animação/Apatia & Autoconceito & Humor & \\
\hline \multirow[t]{2}{*}{ Casadas } & 2,35 & 2,63 & 2,59 & 2,20 & \\
\hline & $(0,57)$ & $(0,47)$ & $(0,56)$ & $(0,67)$ & \\
\hline \multirow[t]{2}{*}{ Descasadas } & 2,13 & 2,36 & 2,52 & 1,93 & \\
\hline & $(0,58)$ & $(0,58)$ & $(0,64)$ & $(0,66)$ & \\
\hline $\begin{array}{l}\text { (b) Escala Locus de Controle } \\
\text { Parental }\end{array}$ & Eficácia parental & Controle parental & Incompetência parental & $\begin{array}{c}\text { Crença na } \\
\text { Sorte ou Destino }\end{array}$ & $\begin{array}{l}\text { Responsabilidade } \\
\text { parental }\end{array}$ \\
\hline \multirow[t]{2}{*}{ Casadas } & 4,14 & 3,54 & 3,45 & 3,35 & 3,26 \\
\hline & $(0,54)$ & $(0,69)$ & $(0,57)$ & $(0,61)$ & $(0,61)$ \\
\hline \multirow[t]{2}{*}{ Descasadas } & 3,83 & 3,35 & 3,38 & 3,17 & 3,22 \\
\hline & $(0,68)$ & $(0,74)$ & $(0,62)$ & $(0,56)$ & $(0,56)$ \\
\hline $\begin{array}{l}\text { Percepção do (a) Relacionamento } \\
\text { Mãe-Filho }\end{array}$ & $\begin{array}{l}\text { Vida profissional } \\
\text { da mãe }\end{array}$ & Preocupações maternais & Sentimento maternos & Futuro materno & $\begin{array}{l}\text { Pontos positivos } \\
\text { do filho adolescente }\end{array}$ \\
\hline \multirow[t]{2}{*}{ Casadas } & 1,87 & 1,73 & 2,16 & 2,25 & 2,40 \\
\hline & $(0,82)$ & $(0,83)$ & $(0,71)$ & $(0,72)$ & $(0,69)$ \\
\hline \multirow[t]{2}{*}{ Descasadas } & 1,61 & 1,52 & 2,04 & 2,04 & 2,20 \\
\hline & $(0,86)$ & $(0,97)$ & $(0,77)$ & $(0,76)$ & $(0,91)$ \\
\hline
\end{tabular}

Nota:

(a) Quanto maior a média, mais positiva a posição do grupo no fator. Valor máximo $=3$.

(b) Quanto maior a média, mais positiva a posição do grupo no fator. Escala; 1 = concordo totalmente; $5=$ discordo totalmente.

Os desvios-padrão encontram-se dentro dos parênteses.

45,38; $p<0,001]$. Não houve efeitos de interação entre estas duas variáveis independentes. As mulheres casadas novamente apresentaram médias mais elevadas que as mulheres não casadas nos fatores eficácia parental, controle parental, incompetência parental, crença dos pais na sorte ou destino e responsabilidade parental. $\mathrm{O}$ grupo das mulheres descasadas revelou sentir-se menos competente e com menos controle nos relacionamentos com seus filhos que as mulheres casadas.

Quanto aos fatores de primeira ordem do locus de controle parental, as maiores médias foram as da dimensão eficácia parental $(\mathrm{x}=4,0)$ seguida pelo controle parental $(\mathrm{x}=$ $3,4)$ e incompetência parental $(x=3,4)$ com médias bem próximas e crença na sorte ou destino $(\mathrm{x}=3,3)$ e responsabilidade parental $(\mathrm{x}=3,2)$.

\section{Percepção e avaliação do relacionamento mãe-filho adolescente}

Foi calculado um índice geral representativo da posição de cada participante quanto a sua percepção sobre o seu relacionamento com seu filho(a) adolescente. Além desse índice geral, foram calculados cinco itens específicos (ver terço inferior da Tabela 5): (1) Vida Profissional (relativo a influência do filho adolescente na vida profissional da mãe); (2) Preocupações Maternas (principais preocupações como mãe de adolescente); (3) Sentimentos Maternos (sentimentos para com o filho adolescente); (4) Próprio Futuro (expectativas da mãe quanto ao próprio futuro) e (5) Pontos Positivos (aspectos positivos de ter filho adolescente).

A análise de variância mista, com medidas repetidas, entre o estado civil e os índices da percepção e avaliação do relacionamento mãe-filho adolescente, revelou a existência de diferenças significativas para o estado civil $\left[F_{(1,254)}=5,85 ; p\right.$ $<0,05]$ e a percepção e avaliação do relacionamento mãefilho $\left[F_{(14)}=38,82 ; p<0,00\right]$. Mais uma vez, não ocorreu um efeito significativo de interação entre as variáveis independentes.

As mulheres casadas apresentaram médias mais elevadas que as mulheres descasadas nas variáveis vida profissional (refletindo a percepção de uma menor interferência do adolescente na sua vida profissional), preocupações maternas (refletindo menor número de preocupações com o filho(a)), sentimentos maternos (refletindo sentimentos de mais satisfação), futuro materno (refletindo uma perspectiva mais otimista quanto ao futuro) e pontos positivos (refletindo maior número de aspectos positivos no ser mãe de filho adolescente). Considerando apenas as cinco dimensões da variável percepção do relacionamento mãe-filho - ignorando o estado civil das respondentes - a maior média ficou com a dimensão pontos positivos de ter filhos adolescentes $(\overline{\mathrm{x}}=2,30)$ e a mais baixa, com a dimensão preocupação materna $(\overline{\mathrm{x}}=1,6)$. As outras dimensões assumiram valores intermediários e bem próximos a estes valores (vida profissional $\overline{\mathrm{X}}=1,7$; sentimentos maternos $\overline{\mathrm{x}}=2,1$; futuro materno $\overline{\mathrm{x}}$ $=2,1)$.

\section{Discussão}

Entre as tarefas com as quais os adultos se defrontam, está a de ajudar os filhos adolescentes a se tornarem adultos felizes e responsáveis (Rosa, 1982). Estes, por sua vez, aumentam as preocupações dos pais, podendo trazer conseqüências desastrosas para o seu bem-estar. A percepção que os pais desenvolvem dos seus filhos adolescentes é influenciada, por um lado, pelo comportamento objetivo do adoles- 
cente - com todas as suas características de transição e afirmação - e, por outro lado, por características dos pais tais como, o bem-estar psicológico, profissional e conjugal. Algumas características psicológicas, como o locus de controle parental, estão claramente envolvidas no relacionamento com os filhos, quer adolescentes ou não.

Face à relevância da família para o bem-estar psicológico dos seus membros, especialmente na fase dos filhos adolescentes, que pode ser sentida por alguns pais como muito difícil, é importante que se procure identificar algumas das características dos pais que estejam relacionadas com o tipo de percepção que se tem do relacionamento com o filho adolescente.

Neste estudo, diferenças entre as mães, quanto à satisfação com a vida e o locus de controle parental, foram preditivas da percepção do relacionamento com o(a) filho(a) adolescente, após as diferenças nas variáveis demográficas terem sido eliminadas estatisticamente. A satisfação com a vida e o locus de controle parental permitiram predições tanto em relação à percepção do relacionamento em geral com o filho adolescente, quanto à percepção de aspectos específicos desse relacionamento. índices gerais de satisfação com a vida e locus de controle parental estiveram associados com a percepção da influência do filho na vida profissional, nos sentimentos maternos para com esta relação e na percepção de pontos positivos em ser mãe de adolescente.

A associação entre os índices gerais destes conjuntos de variáveis foi positiva. A indicação de uma maior satisfação com a vida e de um maior controle interno sobre o comportamento do filho adolescente previu, significativamente, a percepção de uma influência mais positiva do filho na vida profissional da mãe, mais sentimentos positivos com relação a esta relação e a percepção de mais pontos positivos na experiência de ser mãe de filho adolescente.

A incapacidade de controlar o comportamento do filho é um dos motivos de estresse no desempenho do papel materno (Stephens, Franks \& Townsend, 1994). De fato, considerando-se apenas os índices específicos das principais variáveis analisadas, verificou-se que os diversos aspectos do locus de controle parental foram mais preditivos que os aspectos da satisfação com a vida. $\mathrm{O}$ autoconceito (única dimensão da satisfação com a vida com resultados significativos), a eficácia parental, o controle parental e a responsabilidade parental foram os mais significativamente responsáveis pelos resultados gerais. A percepção da chegada da velhice, do desenvolvimento de uma maior eficácia em lidar com o filho, de um maior controle sobre o comportamento do filho e de uma maior responsabilidade por seus comportamentos foram os aspectos específicos envolvidos nas relações entre satisfação com a vida, locus de controle parental e relacionamento mãe-filho.

A maioria das dimensões da satisfação com a vida (congruência, animação vs. apatia e humor), bem como duas outras dimensões do locus de controle parental (incompetência parental e crença na sorte ou destino) não obtiveram índices significativos na participação da previsão da percepção do relacionamento mãe-filho. Estas dimensões, entre- tanto, permitem, conjuntamente, prever significativamente alguns dos aspectos da percepção da relação mãe-filho. Estudos posteriores devem procurar investigar melhor quais os reais componentes dessas dimensões, possíveis responsáveis pela capacidade preditiva conjunta. Algumas evidências apontam para a necessidade de mais investigações quanto à verdadeira estrutura fatorial subjacente aos construtos satisfação com a vida e locus de controle parental. Campis e cols. (1986), por exemplo, também verificaram que o fator crença na sorte ou destino não contribuiu significativamente nas suas análises, sendo então sugerida a sua eliminação. Já o fator incompetência parental surgiu apenas na análise fatorial realizada por Dias e cols. (1996), não existindo na escala de locus de controle parental original.

Quanto às características demográficas da amostra estudada, embora a intenção inicial tenha sido a de controlá-las estatisticamente, verificou-se que o estado civil (casadas vs. descasadas), o tempo de casamento e o número de filhos revelaram associações significativas com algumas dimensões do relacionamento mãe-filho. O estado civil apresentou uma relação positiva com os sentimentos maternos, expectativas quanto ao próprio futuro e vida profissional da mãe. Novamente, as casadas tenderam a ter uma percepção mais positiva que as descasadas nestes índices. Já o número de filhos também previu significativamente a quantidade de pontos positivos em ter-se filho adolescente. Quanto mais filhos, mais positiva esta relação.

O tempo de casamento foi a única característica a apresentar uma associação negativa com algum aspecto do relacionamento mãe-filho: quanto mais tempo de casamento, menos positivos os sentimentos maternos sobre a relação existente com o filho adolescente. Aparentemente, estes últimos resultados estão em desacordo com levantamentos que mostram que, com o passar do tempo, os casais estão mais bem equipados psicológica e financeiramente para lidar com as demandas do casamento e da paternidade (Umberson, 1989). Uma vez superadas as crises do início do casamento, há uma tendência para que os casais se acomodem um ao outro e desenvolvam maior harmonia entre si (Olson \& cols., 1989). Talvez, exatamente por isto, o relacionamento com o filho adolescente, com suas dificuldades características, seja visto de forma mais negativa nos casais mais velhos. Com o passar do tempo, os casais podem ficar mais bem equipados para lidar com os problemas e ajustamentos normais e usuais da vida em família. Talvez fiquem menos tolerantes e pacientes para problemas não normais e usuais, tais como os trazidos pelo filho adolescente. Estabilidade, tranqüilidade e harmonia na família passam a ser bem mais valorizados e promovidos nos casais mais velhos.

Indicações adicionais mostraram que as mulheres descasadas formam um grupo com características um pouco distintas das casadas. Isto é, as descasadas estão sempre um pouco abaixo das casadas em quase todos os índices mensurados. As mulheres casadas apresentam maior consciência da idade e do envelhecimento, indicam que isto as aborrece bem menos, não estão muito animadas com o que fizeram e conseguiram até então, mas estão bem mais conformadas 
que as descasadas. Concordam que não mudariam o passado, pois já obtiveram o que queriam, e acham que estão vivendo os melhores anos de suas vidas. Comparando com as descasadas, o grupo das mulheres casadas demonstrou uma clara acomodação e conformismo com a situação na qual se encontram.

A menor satisfação com a vida demonstrada pelas mulheres descasadas também tem sido observada por outros pesquisadores (p.ex. Anderson, 1977; Larson, 1978; Bengtson \& cols., 1990). O casamento tem sido descrito como uma das principais fontes de satisfação para o ser humano (Bengtson \& cols.; Glenn \& Weaver, 1981; Wilson, 1967). As mulheres que criam seus filhos sozinhas são mais tensas, preocupadas e sujeitas a pressões econômicas e psicológicas (McLanahan \& Adams, 1987; Umberson, 1989).

As casadas também sentem-se mais efetivas, mais capazes de maior controle sobre o filho adolescente e atribuem mais à própria competência os problemas que surgem. Estes resultados acompanham as evidências de que as mulheres que criam seus filhos sozinhas exercem menos controle sobre os mesmos, sendo menos efetivas, principalmente com relação à disciplina (Dornsbuch, Carlsmith, Bushwall, Ritter, Leiderman, Hastorf \& Gross, 1985; Steinberg, 1987).

Quanto ao relacionamento mãe-filho, as mulheres casadas também percebem um relacionamento melhor que as mulheres descasadas. Isto tem sido verificado em outras pesquisas, onde as mulheres casadas apresentam melhor relacionamento com o filho adolescente, enquanto que as mulheres descasadas apresentam baixa satisfação com o papel parental (McLanahan \& Adams, 1987). Paralelamente, Umberson (1989) constatou que o status conjugal divorciado está associado a um alto nível de estresse psicológico e financeiro, prejudicando tanto o relacionamento com os filhos, quanto o bem-estar dos pais.

De modo geral, estes resultados mostram claramente que o envolvimento, o assumir responsabilidades, a percepção de que se é efetiva e responsável pelo que ocorre com o filho adolescente são elementos fundamentais na formação da percepção da qualidade do relacionamento mãe-filho. Até certo ponto, mães que estão mais conscientes do próprio envelhecimento, que já tiveram outros filhos, ainda estão casadas e sentem-se mais eficazes. Com o comportamento do filho sob controle, percebem, de forma mais positiva, seus relacionamentos com os filhos adolescentes.

Reconhece-se, entretanto, que a expressão relacionamento mãe-filho adolescente é bastante ampla para traduzir toda a gama de variáveis que implica. O relacionamento de cada figura parental com o adolescente de cada sexo, a idade do adolescente, a presença de outros filhos, o trabalho, o rendimento dos pais, a relação existente entre o casal são algumas delas.

Hoje já há uma compreensão maior de que a adolescência é um longo período de transição que passa por fases com características próprias. Algumas denominações já surgiram, procurando delimitar a "adolescência inicial" (Steinberg \& Silverberg, 1986; Silverberg \& Steinberg, 1990) e a "adolescência final" (Moore, 1987).
Neste estudo, foi levantada apenas uma pequena parte da questão. Devido à sua complexidade, mais pesquisas são necessárias para trazer novas contribuições ao tema. Pesquisas que definam melhor a verdadeira natureza dos construtos envolvidos e o desenvolvimento de instrumentos mais eficazes. E, especialmente, pesquisas longitudinais, onde se possa tentar estabelecer algum tipo de direção de causalidade entre as variáveis pertinentes ao relacionamento com o filho adolescente.

\section{Referências}

Abbot, D. \& Brody, G. (1985) The relation of child age, gender, and number of children to the marital adjustment of wives. Journal of Marriage and the Family, 47, 77-84.

Adams, D. (1969). Analysis of a Life Satisfaction Index. Journal of Gerontology, 24, 470- 474.

Anderson, M.Q. (1977). A study of the relationship between life satisfaction and self concept, locus of control, satisfaction with primary relationships and satisfaction with work. Dissertation Abstract International, 38, 2638-9A.

Anderson, S.A., Russell, C.S. \& Schumm, W.R. (1983). Perceived marital quality and family life cycle categories: A further analysis. Journal of Marriage and the Family, 45, 227-239.

Bengtson, V., Rosenthal, C. \& Burton, L. (1990). Families and aging: diversity and heterogeneity. Em R.H. Binstock \& L.K. George (Orgs.), Handbook of aging and the social sciences. (Cap. 14, pp. 263-287). New York: Academic Press Inc.

Brehm, S. (1985). Intimate relationships. New York: Random House.

Campis, L.K., Lyman, R.D. \& Prentice-Dunn, S. (1986). The parental locus of control scale: Development and validation. Journal of Clinical Child Psychology, 15, 260-267.

Dias, C.M.S.B. (1995). Relacionamento com filho adolescente: percepção de mães. Tese de Doutorado, Universidade de Brasília, Brasília.

Dias, C.M.S.B., Tróccoli, B.T. \& Dias, M.R. (1996). Padrões psicométricos da Escala de Locus de Controle Parental - PLOC. Em Sociedade Brasileira de Psicologia (Org.), Resumos de Comunicações Científicas, XXVI Reunião Anual de Psicologia (p. 139). Ribeirão Preto: SBP.

Diener, E. (1984). Subjective well-being. Psychological Bulletin, 95, 542-575.

Dornsbuch, S.M. (1989). The sociology of adolescence. Annual Review of Sociology, J5, 233-259.

Dornsbuch, S., Carlsmith, J., Bushwall, S., Ritter, P., Leiderman, H., Hastorf, A. \& Gross, R. (1985). Single parents, extended households and the control of adolescents. Child Development, 56,326-341.

Fasick, F.A. (1984). Parents, peers, youth culture and autonomy on adolescence. Adolescence, 19, 143-157.

Fleming, M. (1993). Adolescência: a autonomia, o desenvolvimento psicológico e a relação com os pais. Porto: Afrontamento.

Furham, A. (1995). A Parental Locus of Control Scale. Manuscrito. Gecas, V. \& Seff, M. (1990). Families and adolescents: A review of the 1980s. Journal of Marriage and the Family, 52, 941-958. 
Glenn, N. \& Weaver, C. (1981). The contribution of marital happiness to global happiness. Journal of Marriage and the Family, 2, 161-169.

Glenn, N. \& McLanahan, S. (1982). Children and marital happiness: A further specification of the relationship. Journal of Marriage and the Family, 2, 63-73.

Greenberg, M., Siegel, J. \& Leitch, C. (1982). The nature and importance of attachment relationships to parents and peers during adolescence. Journal of Youth and Adolescence, 12, 373386.

Harris, R.L., Ellicott, A.M. \& Holmes, D.S. (1986). The timing of psychosocial transitions and changes in women's lives: An examination of women aged 45 to 60. Journal of Personality and Social Psychology, 57,409-416.

Hoyt, D.R. \& Creech, J.C. (1983). The Life Satisfaction Index: A methodological and theoretical critique. Journal of Gerontology, 38, 111-116.

IBGE (1991). Censo demográfico. Rio de Janeiro, número 1.

Koski, K. \& Steinberg, L. (1990). Parenting satisfaction of mothers during midlife. Journal of Youth and Adolescence, 19,465474.

Larson, R. (1978). Thirty years of research on the subjective wellbeing of older Americans. Journal of Gerontology, 33, 109125 .

Lefcourt, H.M. (1991). Locus of control. Em J. Robinson, P.R. Shaver \& L. Wrightsman (Orgs.), Measures of personality and social psychological attitudes (Cap. 9, pp. 413-493). New York: Academic Press.

Lohmann, N. (1980). A factor analysis of life satisfaction: Adjustment and morale measures with elderly adults. International Journal of Aging and Human Development, 11, 35-43.

Lopes, R. (1994). O contexto familiar no desenvolvimento da autonomia e da moralidade na adolescência. Psicologia: Reflexão e Crítica, 7, 59- 74.

MacDermid, S.M. (1990). Developmental interdependencies between parents and adolescents: Links between midlife and adolescent. Dissertation Abstracts International, 51, 3159-3160 B.

McLanahan, S. \& Adams, S. (1987). Parenthood and psychological well-being. Annual Review of Sociology, 13, 237-257.

Montemayor, R. \& Brownlee, J. (1987). Fathers, mothers and adolescents: Gender-based differences in parental roles during adolescence. Journal of Youth and Adolescence, 16, 281-291.

Moore, D. (1987). Parent-adolescent separation: The construction of adulthood by late adolescents. Developmental Psychology, 23, 298-307.
Morgan, S. \& Rindfuss, R. (1985). Marital disruption. American Journal of Sociology, 90, 1055-1077.

Neugarten, B.L., Havighurst, R.J. \& Tobin, S. (1961). The measurement of life satisfaction. Journal of Gerontology, 16, 134143.

Olson, D.H., McCubbin, H., Barnes, H., Larson, A., Muxen, M. \& Wilson, M. (1989). Families: What makes them work? Los Angeles: Sage Publications.

Ribeiro, M.A. (1992). Relações familiares: a percepção dos filhos adolescentes. Estudos de Psicologia, 9, 27-42.

Rollins, B.C. \& Cannon, K.L. (1974). Marital satisfaction over the family life cycle. A revaluation. Journal of Marriage and the Family, 36, 271-282.

Rollins, B.C. \& Feldman, H. (1970). Marital satisfaction over the family life cycle. Journal of Marriage and the Family, 32, 2027.

Rosa, M. (1982). Psicologia da vida adulta. Petrópolis: Vozes.

Sampaio, D. (1994). Vozes e ruídos, diálogos com adolescentes. Lisboa: Caminho.

Steinberg, L. (1987). Single parents, stepparents and the susceptibility of adolescents to antisocial peer pressure. Child Development, 58, 269-275.

Steinberg, L. \& Silverberg, S. (1986). The vicissitudes of autonomy in early adolescence. Child Development, 57, 841-851.

Steinberg, S. \& Steinberg, L. (1990). Psychological well-being of parents with early adolescent children. Development Psychology, 26, 658-666.

Stephens, M., Franks, M. \& Townsend, A. (1994). Stress and rewards in women's multiple roles: The case of women in the middle. Psychology and Aging, 9, 45-52.

Tamir, L. \& Antonucci, T. (1981). Self-perception, motivation and social support through the family life course. Journal of Marriage and the Family, February, 151-161.

Troll, L.E. (1975). Early and middle adulthood. Monterey, California: Brooks/Cole.

Umberson, D. (1989). Relationships with children: Explaining parent's psychological well-being. Journal of Marriage and the Family, 57,999-1012.

Waite, L., Haggstrom, G. \& Kanouse, D. (1985). The consequences of parenthood of the marital stability of young adults. American Sociological Review, 50, 850-857.

Waldemar, J.O. (1983). Quão perturbado é o adolescente "normal"? Revista de Psiquiatria do Rio Grande do Sul, 5, 31-36.

Wilson, W.R. (1967). Correlates of avowed happiness. Psychological Bulletin, 67, 294-306. 